HEP/123-qed

\title{
Nontopological thermal solitons in isotropic ferromagnetic lattices
}

\author{
N. Theodorakopoulos \\ Theoretical and Physical Chemistry Institute \\ National Hellenic Research Foundation \\ Vas. Constantinou 48, GR - 11635 ATHENS, Greece
}

\begin{abstract}
The paper deals with the properties of thermally excited solitons of the isotropic spin- $S$ ferromagnetic chain with nearest-neighbor logarithmic interactions. The exact statistical mechanics of the interacting soliton gas is developed for the general case (arbitrary $S$, temperature and magnetic field). At low temperatures the model's thermodynamics coincides with that of the Heisenberg model. We present analytical approximations of the leading-order asymptotic behavior of the energy in three limiting cases: (a) zero field, low temperature, classical limit; (b) zero field, $T \rightarrow 0, S$ finite (quantum limit); (c) zero field, high temperature, classical limit. Cases (a) and (c) are examples of a dense gas of [non-topological] solitons; results are in agreement with those obtained by the transfer integral method. Case (b) illustrates the behavior of a dilute, yet strongly interacting soliton gas; results for the thermodynamics are very close to (but not identical with) spin-wave and/or Bethe-Ansatz predictions.
\end{abstract}

75.10.Hk, 75.10.Jm, 05.20.-y, 52.35.Sb

Typeset using REVTEX 


\section{INTRODUCTION}

Theoretical and experimental work with one dimensional magnets has provided ample evidence for the existence and importance of solitons [1]. The development of solitonic concepts and techniques has been characterized by a remarkable degree of cross-fertilization between statistical physics, field theory and applied mathematics. Indeed, much of our present understanding of the thermal behavior of magnetic solitons results from Mikeska's seminal exploitation [2] of the formal equivalence between the anisotropic Heisenberg model (with an external field) and the Sine-Gordon continuum. There has been no lack of "direct" candidates from magnetism. The isotropic Heisenberg ferromagnet (IHF) has been known to be integrable in the continuum limit [3, [4], and a variant, the Ishimori-Haldane-Fadeev ferromagnet (IHFF) is completely integrable on a lattice [5]7. In both models, the properties of solitons at thermal equilibrium, although of fundamental interest, are difficult to extract. The reason for this is twofold: (i) the presence of non-topological solitons, whose amplitudes can become arbitrarily small and their numbers presumably arbitrarily large. (ii) the fact that those non-topological solitons, which perform breather-like internal oscillations in addition to their translational motion, actually reduce to linear spin waves in the small amplitude limit. The first problem is generic to all systems which carry non-topological solitons (e.g. the Toda lattice [8]); it means that one must incorporate interactions between solitons in order to achieve a valid description of their statistics at any finite temperature [9]. The second problem has been dealt with in the case of Sine-Gordon breathers. There, it has been demonstrated (in leading order asymptotics [10]) that if one allows the presence of both breathers and phonons, enough of either will be displaced to maintain the total number

of degrees of freedom. Double counting can in fact be avoided less tediously a priori: exact formulations of statistical mechanics in terms of either nonlinear phonons [11] or breathers |12 yield identical results.

The present work deals with the thermodynamics of IHFF solitons. At sufficiently low temperatures, the model's properties coincide with those of the IHF. Since the [integrable] 
IHF continuum can be considered to be a good approximation to the IHF lattice under the same condition, i.e., at low temperatures, our results in effect provide a description of soliton statistical mechanics of the IHF as well. This equivalence has been exploited in a recent Letter [13], where many of our key results have appeared without proof [14].

The aims of this paper are: (i) to give a reasonably complete account of the theory of [exact] soliton statistical mechanics in the only magnetic system where this has been possible to a full extent and, (ii) to support the conclusions of the theory by presenting its application to selected analytically tractable limiting cases. Of particular interest in the latter context is the treatment of the high-temperature regime (not reported in Ref. [13]), which validates the concept of a strongly interacting, dense gas of extremely localized solitons.

The paper is organized as follows: Section II introduces the model and its dynamics. It reviews the basic properties of one- and two-soliton solutions and discusses the conservation laws which control the symmetries of soliton/soliton phase shifts and are thus central to the statistical mechanics. Section III formulates the statistical mechanics in terms of occupation probabilities for microstates. Its main result is formulated in terms of a two-dimensional integral equation. It will be shown that the exact thermodynamic quantities (soliton density, energy, magnetization) are controlled by the asymptotic behavior of soliton quasienergies in phase space. Section IV deals with the limiting case of zero-field, high and low-temperature asymptotic behavior. In the latter case, it will be possible to derive results for both the quantum and the classical regime. Concluding remarks are made in Section V. The relationship between thermodynamic properties and integral-equation asymptotics is derived in the Appendix.

\section{DYNAMICS}

\section{A. The model}

The IHFF model Hamiltonian 


$$
H=-2 J S^{2} \sum_{n} \ln \left(\frac{1}{2}+\frac{\vec{S}_{n} \cdot \vec{S}_{n+1}}{2 S^{2}}\right)-g B \sum_{n}\left(S_{n}^{z}-S\right),
$$

describes a chain of $N$ atoms, each of which carries a spin $\vec{S}_{n}$ of length $S$, and is subjected to an external field $B$ along the z-axis. The particular form of the interaction guarantees complete integrability [5] at the classical limit, i.e., as $S \rightarrow \infty, \hbar \rightarrow 0, \hbar S \rightarrow S_{c}, J \rightarrow$ $0, J S^{2} \rightarrow j, g \rightarrow 0, g S \rightarrow g_{c}$ [15]. It will, however, be necessary to keep the value of $S$ as a parameter in the problem, both in order to deal with semiclassical approximations of quantum chains and because the equation which describes statistical mechanics at the classical limit is singular.

The classical dynamics of (2.1) is described by

$$
\frac{d}{d t} \vec{S}_{n}=\frac{\partial H}{\partial \vec{S}_{n}} \times \vec{S}_{n}
$$

where time is measured in units of $\hbar /(J S)=S_{c} / j$. The fact that the dynamical system (2.2) is completely integrable has been demonstrated in a variety of ways. Ref. [5] makes use of the gauge equivalence of the dynamics arising from (2.2) (for $B=0$ ) to the AblowitzLadik(AL) [16] version of the discrete nonlinear Schrödinger equation. Refs. [6,7] conjecture the existence of such a model by considering a sequence of finite- $S$ models which are BetheAnsatz solvable [17]. The complete inverse scattering theory has also been presented [18]. We will follow [5] and make direct reference to [16] when appropriate.

\section{B. Properties of single solitons}

The problem of the magnetic field is disposed of first. The physical effect of the field is to make all spins perform a uniform precessional motion with angular frequency $h=g_{c} B / j$. This motion can be eliminated by introducing a rotating coordinate frame. Now, since the dynamics of the zero-field IHFF (2.2) can be mapped to the the dynamics of the AL model, it is possible to describe any general multi-soliton solution of (2.2) in terms of the corresponding AL solution, and superimpose the uniform precession due to the magnetic field at the end. This simplification is of particular importance for the statistical mechanics, 
since it means that all relevant properties of interactions between solitons are controlled by the values of their dynamical parameters for vanishing magnetic field; it does not imply however that thermodynamic properties have a trivial dependence on the magnetic field ( $c f$. Section III).

The form of the single soliton solution ( $c f$. [5]), demonstrates that all physical soliton properties can be described in terms of two parameters, $w$ and $k$. Thus, the soliton has a spatial extent (in units of the lattice constant $\ell$ ) equal to $1 /(2 w)$, it moves with a translational velocity $U(w, k)=\sinh 2 w \sin 2 k /(2 w)$, and performs an internal oscillation of frequency $\Omega(w, k)=2 k U(w, k)+2(\cosh 2 w \cos 2 k-1)+h$; furthermore, one can evaluate the two obvious integrals of motion, namely, the total magnetization

$$
M(w, k)=-2 \frac{\sinh 2 w}{\cosh 2 w-\cos 2 k},
$$

(in units of $S_{c}$ ), and energy

$$
E(w, k)=8 w-h M(w, k)
$$

(in units of $j$ ) carried by a single soliton. The soliton canonical momentum $P$, defined [19] via $(\partial P / \partial E)_{M}=1 / U$, (in units of $\left.S_{c} / \ell\right)$, determines the correct measure to be used in statistical mechanics:

$$
d P d M=\frac{\partial(P, M)}{\partial(w, k)} d w d k=\frac{8}{U}\left(\frac{\partial M}{\partial k}\right)_{w} d w d k=32 \frac{w}{(\cosh 2 w-\cos 2 k)^{2}} d w d k
$$

In the limit $w \rightarrow 0$, the properties of a soliton characterized by $(w, k)$ reduce to those of a plane spin wave with wavevector $q=2 k$ and frequency $4 \sin ^{2}(q / 2)$.

\section{Multisoliton solutions: soliton-soliton phase shifts}

The simplest way to evaluate the asymptotic space shifts resulting from the interaction of two solitons is to go back to the AL formulation of the inverse-scattering transform, write down the two-soliton solution, and examine its asymptotic form as $t \rightarrow \pm \infty$. The calculation is tedious but straightforward. Here, we only quote the result and make a few comments. 
The phase shift which a soliton $(w, k)$ experiences due to its collision with a soliton $\left(w^{\prime}, k^{\prime}\right)$ is given by

$$
\Delta\left(w, k ; w^{\prime}, k^{\prime}\right)=\frac{1}{2 w} \ln \left[\frac{\cosh 2\left(w^{\prime}+w\right)-\cos 2\left(k-k^{\prime}\right)}{\cosh 2\left(w^{\prime}-w\right)-\cos 2\left(k-k^{\prime}\right)}\right] .
$$

It follows that the sum rule

$$
\int_{0}^{\pi} d k \Delta\left(w, k ; w^{\prime}, k^{\prime}\right)=\frac{2 \pi}{w} \min \left(w, w^{\prime}\right)
$$

is always satisfied. Furthermore, the soliton/soliton phase shifts obey the following symmetry (generalizable to any multisoliton solution characterized by $\left\{w_{\lambda}, k_{\lambda}\right\}$ ):

$$
\sum_{\lambda \neq \lambda^{\prime}} w_{\lambda} \Delta\left(w_{\lambda}, k_{\lambda} ; w_{\lambda^{\prime}}, k_{\lambda^{\prime}}\right)=0
$$

\section{Phase shift symmetries and conservation laws}

Symmetries of the type (2.8) are generic to soliton-bearing systems. They reflect the existence of a low-order integral of motion $I=\sum_{\lambda} w_{\lambda} U_{\lambda}$, (where $U_{\lambda}$ is the velocity of the $\lambda$ th soliton) and imply that the [conserved] quantity $\sum_{\lambda} w_{\lambda}$ travels at a constant speed. Inspection of the formula for the group velocity shows that the required conserved quantity is AL's $C_{1}$, or, in the spin language,

$$
I=\sum_{n} \tilde{\chi}_{n} \equiv \sum_{n} \frac{\vec{S}_{n} \cdot\left(\vec{S}_{n+1} \times \vec{S}_{n-1}\right)}{\left(1+\vec{S}_{n} \cdot \vec{S}_{n+1}\right)\left(1+\vec{S}_{n} \cdot \vec{S}_{n-1}\right)}
$$

and reflects the invariance of the original spin Hamiltonian under infinitesimal rotations of each spin $\vec{S}_{n}$ around an axis defined by the spin-space gradient

$$
\nabla_{\vec{S}_{n}} I \equiv \nabla_{\vec{S}_{n}}\left(\tilde{\chi}_{n+1}+\tilde{\chi}_{n}+\tilde{\chi}_{n-1}\right)
$$

Note that generally (i.e. for $h \neq 0$ ) $w$ is proportional to the exchange part of soliton energy. It is possible to show that the center of exchange energy of the entire IHFF lattice travels at a constant speed, independently of any soliton considerations. Given an infinite chain with any type of isotropic nearest neighbor spin interaction $f\left(\vec{S}_{n} \cdot \vec{S}_{n+1}\right)$, consider the quantity 


$$
X=\frac{1}{Z} \sum_{n} n g\left(\vec{S}_{n} \cdot \vec{S}_{n+1}\right)
$$

where $g$ is such that the denominator $Z=\sum_{n}\left[g\left(\vec{S}_{n} \cdot \vec{S}_{n+1}\right)\right]$ is a constant of the motion. The derivative $d X / d t$ can be written down by using (2.2); it turns out that, in order for it to remain constant under the Hamiltonian flow, (i) $g$ must be proportional to $f$ (and thus $Z$ proportional to the exchange part of the Hamiltonian) and, (ii) the quantity

$$
\sum_{n} g^{\prime}\left(\vec{S}_{n} \cdot \vec{S}_{n+1}\right) g^{\prime}\left(\vec{S}_{n} \cdot \vec{S}_{n-1}\right) \vec{S}_{n} \cdot\left(\vec{S}_{n+1} \times \vec{S}_{n-1}\right)
$$

must be a constant of the motion, as indeed is the case with the logarithmic (but not with the Heisenberg) interaction. Thus, the existence of the constant of motion $I$ guarantees a very special status to the exchange energy of the IHFF model (analogous to that of the mass in a lattice model [8]).

\section{SOLITON THERMODYNAMICS: EXACT RESULTS}

The general part of the formalism used has been presented in [20], in the context of the Sine-Gordon equation; the reader is referred there for details. The physics involve the minimization of the classical free energy functional with respect to the occupation probability of each state. The procedure is self-consistent since the density of states is itself a functional of the occupation probabilities, due to the pairwise additive interactions (2.6) which restrict available phase space. The thermodynamics derived within this framework is in principle exact, with a single caveat: because solitons with a very large characteristic length are in practically indistinguishable from linear excitations (spin waves, $c f$. above) one expects that a correct statistical mechanical treatment will ultimately eliminate one or the other. The situation is analogous with the famous case of the Sine-Gordon breather. It appears therefore expedient to formulate the theory in terms of solitons alone (cf. [21]).

The thermodynamic potential (free energy) of the interacting soliton gas is given by

$$
-\beta F=\int d \Gamma R_{0}(\Gamma) \bar{n}(\Gamma)
$$


where $\beta=J S^{2} / k_{B} T$ is the dimensionless inverse temperature, $\Gamma$ is shorthand for the point $(w, k)$ in the soliton parameter phase space, and the phase space element $d \Gamma$ is defined by the measure (2.5). The density of states $R_{0}(\Gamma)$ refers to the non-interacting soliton gas (with our choice of units ( $c f$. Section IIb) equal to $N /(2 \pi) S^{2}$ ) and the occupation probability $\bar{n}(\Gamma) \equiv \exp (-\beta \epsilon)$ defines a characteristic [field- and temperature-dependent] quasiparticle energy $\epsilon(\Gamma)$. The latter is determined by the integral equation

$$
\beta \epsilon(\Gamma)=\beta E(\Gamma)+\frac{1}{2 \pi} \int d \Gamma^{\prime} \Delta\left(\Gamma^{\prime}, \Gamma\right) e^{-\beta \epsilon\left(\Gamma^{\prime}\right)}
$$

A further quantity of interest is the density of available states at any given point in phase space expressed as fraction of $R_{0}(\Gamma)$, i.e., $\hat{R}(\Gamma) \equiv R(\Gamma) / R_{0}(\Gamma)$. In the IHFF case, equations (3.1) - (3.2) take the form

$$
-\beta f=\frac{16}{\pi} \int_{0}^{\pi} d k \int_{0}^{\infty} d w \frac{w}{(\cosh 2 w-\cos 2 k)^{2}} e^{-\beta \epsilon(w, k)}
$$

and

$$
\begin{aligned}
& \beta \epsilon(w, k)=\beta E(w, k)+\frac{8}{\pi} S^{2} \int_{0}^{\pi} d k \int_{0}^{\infty} d w^{\prime} \quad \frac{1}{\left(\cosh 2 w^{\prime}-\cos 2 k^{\prime}\right)^{2}} \\
& \ln \left[\frac{\cosh 2\left(w^{\prime}+w\right)-\cos 2\left(k-k^{\prime}\right)}{\cosh 2\left(w^{\prime}-w\right)-\cos 2\left(k-k^{\prime}\right)}\right] e^{-\beta \epsilon\left(w^{\prime}, k^{\prime}\right)},
\end{aligned}
$$

respectively, where $f$ is now the free energy per site. Furthermore,

$$
\hat{R}(w, k)=\frac{1}{E_{0}(w)}\left(\frac{\partial \beta \epsilon(w, k)}{\partial \beta}\right)_{\beta h} .
$$

The derivation is straightforward, and involves use of (2.5) and (2.6). Some special attention must be paid to the analogy of (3.5) with Eq.(19) of [20]; in this case, the crucial symmetry refers to the exchange part of the energy of a free soliton ( $c f$. above); hence the denominator $E_{0}(w)=8 w$ in (3.5). Moreover, the derivative with respect to $\beta$ must be taken at constant $\beta h$.

The thermodynamic functions we are interested in, i.e. energy, soliton density and magnetization can be obtained by differentiation of (3.3) with respect to $\beta, \mu$ and $h$ respectively. The soliton chemical potential must vanish, since the soliton density is not a priori fixed. 
The details are presented in the Appendix. The important feature is that we do not require detailed knowledge of the solution of (3.4) at all points in the two-dimensional region defined by $0<w<\infty, 0<k<\pi$. The thermodynamic behavior of the soliton gas is controlled by the asymptotic properties of (3.4) in the limits $w \rightarrow 0$ and $w \rightarrow \infty$. In particular, the free energy is given by

$$
\beta f=-\frac{1}{2 \pi} \int_{0}^{\pi} d k C_{\infty}(k)
$$

and the soliton density per site by

$$
n_{s}=\frac{1}{2 \pi} \int_{0}^{\pi} d k\left[1-\left(\frac{\partial V_{0}}{\partial V_{\infty}}\right)_{\beta h}\right]
$$

where

$$
\begin{gathered}
V(w, k) \equiv \frac{\partial \beta \epsilon(w, k)}{\partial w}, \\
V_{\infty} \equiv \lim _{w \rightarrow \infty} V(w, k)=8 \beta, \\
V_{0} \equiv \lim _{w \rightarrow 0} V(w, k), \text { and } \\
C_{\infty}(k) \equiv \lim _{w \rightarrow \infty}(\beta \epsilon(w, k)-\beta E(w, k)) .
\end{gathered}
$$

It follows that the internal energy $u$ and magnetization $m$ per site can be obtained by direct differentiation of (3.6) with respect to $\beta$ and $h$ respectively, i.e.,

$$
\begin{gathered}
u=\left(\frac{\partial \beta f}{\partial \beta}\right)_{h}=-\frac{1}{2 \pi} \int_{0}^{\pi} d k\left(\frac{\partial C_{\infty}(k)}{\partial \beta}\right)_{h} \\
m=\left(\frac{\partial \beta f}{\partial h}\right)_{\beta}=-\frac{1}{2 \pi} \int_{0}^{\pi} d k\left(\frac{\partial C_{\infty}(k)}{\partial h}\right)_{\beta} .
\end{gathered}
$$




\section{SOLITON THERMODYNAMICS: ANALYTIC APPROXIMATIONS}

\section{A. The classical, zero-field, low temperature case}

At low temperatures we expect thermodynamic properties to be dominated by solitons whose exchange energies are lower than $1 / \beta$, i.e., with parameter values $w \ll 1$. If, in addition, $k \geq w$, it is possible to approximate the exact phase shift (2.6) by

$$
\Delta\left(w, k ; w^{\prime}, k^{\prime}\right)=\frac{2 \pi}{w} \min \left(w, w^{\prime}\right) \delta\left(k-k^{\prime}\right)
$$

Note that the above approximation preserves the symmetry (2.8) of the soliton/soliton phase shift and satisfies the sum rule (2.7), so we can reasonably expect it to exhibit at least the salient features of the exact solution of (3.4). Introducing (4.1) in (3.4), it is possible to decouple the statistics of solitons with different $k$-values, and transform the two-dimensional integral equation (3.4) to a set of one-dimensional integral equations in which $k$ appears as a parameter:

$$
\beta \epsilon(w, k)=\beta E(w, k)+8 S^{2} \int_{0}^{\infty} d w^{\prime} \frac{\min \left(w, w^{\prime}\right)}{\left(w^{\prime 2}+\sin ^{2} k\right)^{2}} e^{-\beta \epsilon\left(w^{\prime}, k\right)}
$$

The integral equation (4.2) can be transformed to a second-order differential equation for $x \equiv \beta \epsilon(w, k):$

$$
\frac{d^{2} x}{d t^{2}}=-8 \frac{e^{-x-\beta h M(t, k)}}{\left(1+a t^{2}\right)^{2}}
$$

where $a=\sin ^{2} k / S^{2}$ and we have used a rescaled independent variable $t=S w / \sin ^{2} k$, in terms of which $M(t, k)=-2 t /\left(1+a t^{2}\right) / S$. Eq. (4.3) must be solved subject to mixed initial and final conditions, i.e., $x(0)=0, d x / d t(\infty)=v_{\infty}=8(\beta / S) \sin ^{2} k$. (Note the rescaling with respect to $V_{\infty}$ of $(\underline{3.810})$

The remainder of this Section specializes to the case $h=0$.

In the classical limit, $S \rightarrow \infty, \beta$ finite, the quantities

$$
v_{\infty}=8 \beta \sin ^{2} k / S
$$


and $a$ become infinitesimally small. Eq. (4.3) can be further simplified to

$$
\frac{d^{2} x}{d t^{2}}=-8 e^{-x}
$$

which, for the given boundary conditions, has the exact solution

$$
x(t)=2 \ln \left\{\frac{4}{v_{\infty}} \sinh \left(\frac{v_{\infty} t}{2}+\sinh ^{-1}\left(\frac{v_{\infty}}{4}\right)\right)\right\} .
$$

Two remarks are in order here. First, that exactly the same equation (4.5) controls the classical limit of the Sine-Gordon breather gas [21]. Second, that if one regards (4.3), with $h=0$, as a mechanical system, (4.5) represents its "autonomous" limit. The asymptotic properties of (4.6) are particularly simple ( $c f$. definitions above):

$$
v_{0}=\sqrt{16+v_{\infty}^{2}},
$$

and

$$
C_{\infty}=2 \ln \left\{\frac{v_{0}+v_{\infty}}{v_{\infty}}\right\}
$$

It follows that

$$
-\frac{\partial C_{\infty}}{\partial v_{\infty}}=2\left(1-\frac{\partial v_{0}}{\partial v_{\infty}}\right) .
$$

In the classical limit under consideration, This means ( $c f$. Eq. (3.7)) that the magnetic lattice will be equally populated with solitons of all $k$, producing a total density of $1 / 2$ solitons per site, as expected from counting the classical degrees of freedom. The internal energy density can be found by inserting (4.9) and (4.4) in (3.9) to be equal to $k_{B} T$, as demanded by the equipartition theorem.

\section{B. The finite $S$, zero-field, $\beta \rightarrow \infty$ limit}

The core of the approximation scheme developed in the preceding subsection remains valid away from the classical limit if the temperatures become extremely low. More precisely, for $S$ finite and $\beta$ large, consider the range of $k$ defined by 


$$
v_{\infty}=8(\beta / S) \sin ^{2} k \leq A
$$

where $A \gg 1$ but finite. As $\beta \rightarrow \infty$, this range is bounded by a characteristic $k_{c} \propto 1 / \sqrt{\beta}$. For such $k$ 's, the parameter $a$ is smaller than $1 / \beta$. In the limit $\beta \rightarrow \infty$ this allows us to substitute (4.3) by (4.5). On the other hand, values of $k$ outside this range are (in the limit $\beta \rightarrow \infty)$ irrelevant. The argument for this is as follows: such $k$-values lead to $v_{\infty} \geq A \gg 1$; numerically large values of $v_{\infty}$ lead in turn to comparably large values of $v_{0}$, in the context of both (4.5) and (4.3). In the former case this can be proved by inspecting (4.7). Moreover, it is in both cases clear that a $v_{\infty} \gg 1$ can only come from a $v_{0} \gg 1$. This however means that $x(t)$ grows very rapidly from its initial value $x(0)=0$. As this happens, the right hand sides of both (4.5) and (4.3) vanish, dominated by the exponential factor. Since the "acceleration" vanishes before it has any time to act, the "velocity" stays at its initial value, i.e., $v_{\infty} \approx v_{0}$

$$
1-\frac{d v_{0}}{d v_{\infty}} \approx 0
$$

and therefore such solitons are not thermally excited $(c f .(3.7))$. As a consequence, in the limit $\beta \rightarrow \infty$, it is legitimate to use (4.5) in lieu of (4.3) for all $k$, and thus exploit the properties (4.7)-(4.9) of the solution (4.6).

Inserting (4.10) in (3.7), and approximating $\sin k$ by its argument, we obtain the leadingorder contribution to the soliton density,

$$
\begin{aligned}
n_{s} & =\frac{1}{\pi} \sqrt{\frac{S}{2 \beta}} \int_{0}^{\infty} d x\left(1-\frac{x^{2}}{\sqrt{1+x^{4}}}\right) \\
& =\frac{\sqrt{2 \pi}}{\left[\Gamma\left(\frac{1}{4}\right)\right]^{2}}\left(\frac{k_{B} T}{J S}\right)^{1 / 2} .
\end{aligned}
$$

The corresponding result for the energy density, obtained by making use of (4.10) in (4.9), is

$$
u=2 \frac{\sqrt{2 \pi}}{\left[\Gamma\left(\frac{1}{4}\right)\right]^{2}} J S\left(\frac{k_{B} T}{J S}\right)^{3 / 2} .
$$

A few remarks are in order here. First, Eq. (4.12) means that the soliton gas is dilute. Not all solitons are excited. This is a characteristic of the quantum regime. In the classical 
limit ( $c f$. previous and following subsection) solitons must exhaust all degrees of freedom. (It should be remembered that no provision for linear excitations has been made.) Second, solitons carry an average energy of $2 k_{B} T$ each, as suggested by equipartition. Numerical solution of (4.3) indicates [13] that this result is only valid in the limit $\beta \rightarrow \infty$. Third, the reason the approximation (4.1) works is that the conditions for its validity are met by thermal solitons; characteristic $w$-values of $1 / \beta$ are much lower than the typical $k$ values of $\mathcal{O}\left(k_{c}\right)$ (quantum case), or $\mathcal{O}(1)$ (classical case, preceding subsection). Fourth, the numerical value of the prefactor of the energy, 0.3814 , is close to the spin-wave result of 0.3684 . Comparable results have been obtained in the $S=1 / 2$ case in terms of the Bethe-Ansatz 22,23]. In view of the soliton-magnon duality at low amplitudes, this is not unexpected.

\section{The classical, zero-field, high temperature limit}

In the high temperature regime, $\beta<1$, narrow solitons which carry large amounts of exchange energy, i.e., with values of $w>1$ become predominant. As long as the characteristic parameters $w, w^{\prime}$ of two such solitons do not come very close to each other, the hyperbolic cosines in the exact soliton/soliton phase shift (2.6) can be approximated by exponentials, leading to

$$
\Delta\left(w, k ; w^{\prime}, k^{\prime}\right)=\frac{2}{w} \min \left(w, w^{\prime}\right)
$$

The above approximation is the exact opposite of (4.1): instead of a $\delta$ function in $k$-space, which decouples soliton modes corresponding to different $k$ 's, (4.14) is independent of $k, k^{\prime}$, ensuring that all soliton modes couple to each other. It should be further noted that (4.14) satisfies the sum rule (2.7), just like the low- $T$ approxination (4.1).

Substitution of (4.14) in (3.4) yields

$$
\beta \epsilon(w, k)=8 \beta w+\frac{128 S^{2}}{\pi} \int_{0}^{\pi} d k^{\prime} \int_{w_{0}}^{\infty} d w^{\prime} e^{-4 w^{\prime}} \min \left(w, w^{\prime}\right) e^{-\beta \epsilon\left(w^{\prime}, k^{\prime}\right)},
$$

where again the hyperbolic cosine in the denominator of (3.4) has been approximated by an exponential, to ensure consistency with (4.14). It should be emphasized that these approx- 
imations are crude, and that they cannot be expected to yield accurate results for values of $w \ll 1$. The point is that such low energy solitons are irrelevant at high temperatures.

In accordance with this argument, we temporarily exclude soliton modes with very low values of $w$ from any further consideration by introducing a lower cutoff in (4.15). This will be useful in controlling singularities, although the value of the cutoff will be set to zero at the end of this calculation.

Since the right-hand side of (4.15) is $k$-independent, $\epsilon$ can only depend on $w$; Integration over $k^{\prime}$ can be performed trivially, and reduction to an o.d.e. follows the steps of the previous subsection. The result is

$$
\frac{d^{2} x}{d w^{2}}=-128 S^{2} e^{-(x+4 w)} .
$$

The most general solution of Eq. (4.16) consistent with the condition $\lim _{w \rightarrow \infty} d x / d w \equiv$ $V_{\infty}=8 \beta$ is

$$
x(w)=2 \ln \left\{\frac{4}{\lambda} e^{-2 w} S \sinh (2 \lambda w+b)\right\},
$$

where $\lambda=1+2 \beta$. We do not know the value of $b$, since we have no knowledge of the exact behavior of $x(w)$ as $w \rightarrow 0$. The following procedure will be adopted instead: $b$ will be set equal to zero, and the value of the cutoff will be fixed by demanding $x\left(w_{0}\right)=0$. This leads to $w_{0}=1 /(8 S)$, which vanishes at the classical limit. The solution (4.17) has the asymptotic properties

$$
V_{0}^{(S)} \equiv V\left(\frac{1}{8 S}\right)=-4+16 S \quad(S \gg 1)
$$

and

$$
C_{\infty}=-2 \ln \left(\frac{1+2 \beta}{2}\right)+2 \ln S .
$$

Both (4.18) and (4.19) are valid for all $k$. In the limit $S \rightarrow \infty, V_{0}^{(S)}$ diverges, but $\lim _{S \rightarrow \infty} d V_{0}^{(S)} / d V_{\infty}=0$, and thus, from (3.7) the soliton density must equal $1 / 2$. Furthermore, from (3.9) and (4.19), the internal energy per site 


$$
u=\frac{2 j}{1+2 j /\left(k_{B} T\right)}
$$

can be calculated, in agreement with the exact result obtained by the transfer integral method [24].

Although this completes the derivation of soliton thermodynamics at the classical high temperature limit, it is instructive to examine the basic features of the analytical solution (4.17). For example, using (4.17) in the alternative definition of the soliton density ( $c f$. Ref. [20]), allows us to examine the $w$-distribution of thermal solitons (with the understanding that no conclusions should be drawn about details in the region $0<w \ll 1$ ):

$$
n_{s}=2 \lambda \int_{0}^{\infty} d w \frac{1}{\sinh ^{2}(2 \lambda w)}\left\{\frac{2 \lambda w}{\tanh (2 \lambda w)}-1\right\} .
$$

Eq. (4.21) implies that solitons which are thermally excited have values of $w \approx 1 / \lambda$. In other words, typical thermal solitons cannot become narrower than a lattice constant. Since each such soliton carries an energy of order $j$, the total energy per site is of order unity at high temperatures, as expressed by (4.20).

\section{CONCLUDING REMARKS}

We have presented an "advanced phenomenology" of the interacting soliton gas for an isotropic ferromagnetic chain. The theory is in principle exact but requires solving a twodimensional integral equation, i.e. in effect has the same degree of complexity as the quantum mechanical Bethe-Ansatz. In the case of vanishing magnetic field it has been possible to develop valid approximations in the high and low temperature regime and derive analytic expressions for thermodynamic quantities. The results presented provide detailed information about the manner in which nonlinear modes accommodate themselves at finite temperatures.

In the low temperature regime, the fortunate occurrence of a gapless spectrum allows a semiquantitative understanding of the quantum, $T \rightarrow 0$, limit of the Heisenberg chain with essentially classical techniques. The behavior of the soliton density suggests the presence 
of a "dilute" soliton gas. The quotation marks imply that the term should be used with some caution. It refers to typical distance between the centers of two solitons. At low temperatures, this is indeed large ( of order $T^{-1 / 2}$ ); however, the spatial extent of a thermal soliton is of order $1 / T$, i.e., much larger. There is therefore significant overlap between solitons, suggesting the presence of strong interactions. It is interesting to digress at this point and consider the analogous situation in the Toda lattice. There, the typical intersoliton distance at low temperature is of the same order as the spatial extent of thermal solitons, i.e., $T^{-1 / 3}$.

It appears that a properly formulated soliton phenomenology has a range of validity which far exceeds the limits imposed by the original conceptual framework [25,26]. In the particular case of the quantum low-temperature regime, marginal uncertainties in the BetheAnsatz make it presently impossible to decide whether the soliton result is asymptotically exact. It is however clear, that soliton theory presents a better alternative to spin waves: the magnetization obtained from the soliton theory vanishes at any finite temperatures $(c f$. the divergent magnetization obtained from spin-wave theory).

At high temperatures it appears, that in spite of the crudeness of our approximation, the theory again describes leading order corrections. The exact agreement of (4.20) with the transfer integral result should be regarded as fortuitous. In this case the soliton gas is dense in the traditional sense. Intersoliton distances and soliton spatial extent are both in the range of the lattice constant.

\section{APPENDIX:}

In order to derive (3.6), we subtract the "bare" part of the soliton energy, $\beta E(w, k)$ from both sides of (3.4) and integrate both sides over all $k$. The result is

$$
\int_{0}^{\pi} d k\{\beta \epsilon-\beta E(w, k)\}=8 S^{2} \int_{0}^{\pi} d k \int_{0}^{\infty} d w^{\prime} \frac{2}{\left(\cosh 2 w^{\prime}-\cos 2 k^{\prime}\right)^{2}} 2 \min \left(w, w^{\prime}\right) e^{-\beta \epsilon\left(w^{\prime}, k^{\prime}\right)},
$$


where we have made use of the sum rule (2.7). In the limit $w \rightarrow \infty$, the integrand of the left-hand side of (A1) is $C_{\infty}$, whereas the right-hand side coincides, except for a prefactor $1 /(2 \pi)$, with $-\beta f(c f$. Eq. 3.3). This proves (3.6).

In order to derive (3.7), we differentiate both sides of (3.4) with respect to $w$. In the limit $w \rightarrow 0$, this yields

$V_{0}(k)=V_{\infty}+\frac{4 \beta h}{1-\cos 2 k}+\frac{8 S^{2}}{\pi} \int_{0}^{\pi} d k \int_{0}^{\infty} d w^{\prime} \frac{1}{\left(\cosh 2 w^{\prime}-\cos 2 k^{\prime}\right)^{2}} \frac{4 \sinh 2 w^{\prime}}{\cosh 2 w^{\prime}-\cos 2\left(k^{\prime}-k\right)} e^{-\beta \epsilon\left(w^{\prime}, k^{\prime}\right)}$.

Differentiating both sides of (A2) with respect to $V_{\infty}$ at constant $\beta$, we obtain

$$
\left(\frac{\partial V_{0}(k)}{\partial V_{\infty}}\right)_{\beta h}=1-\frac{8 S^{2}}{\pi} \int_{0}^{\pi} d k \int_{0}^{\infty} d w^{\prime} \frac{8 w^{\prime}}{\left(\cosh 2 w^{\prime}-\cos 2 k^{\prime}\right)^{2}} \frac{4 \sinh 2 w^{\prime}}{\cosh 2 w^{\prime}-\cos 2\left(k^{\prime}-k\right)} \hat{R}\left(w^{\prime}, k^{\prime}\right) e^{-\beta \epsilon\left(w^{\prime}, k^{\prime}\right)}
$$

where we have used (3.5) for the density of states $\hat{R}$. Eq. 3.7 can now be obtained by integrating both sides of (A3) over $k$.

A further by-product of (A3) is that, in the limit $k \rightarrow 0$, the integrand in the right-hand side expresses the total magnetization in soliton component form. This implies that the total magnetization per site (in units of $S_{c}$ ) can be alternatively expressed in terms of the asymptotic properties of (3.4), as

$$
m=\lim _{k \rightarrow 0}\left(\frac{\partial V_{0}(k)}{\partial V_{\infty}}\right)_{\beta h}
$$

The above alternative can be useful in situations where the integral (3.10) cannot be calculated exactly. In all limiting situations which we have treated analytically ( $c f$. Section IV), (A4) guarantees a vanishing magnetization. 


\section{REFERENCES}

[1] H.J. Mikeska and M. Steiner, Adv. Phys. 40, 191 (1991)

[2] H.J. Mikeska, J. Phys. C 11, L29 (1980)

[3] L.A. Takhtajan, Phys. Lett. A 64, 235 (1977)

[4] H.C. Fogedby, J. Phys. A 13, 1467 (1980)

[5] Y. Ishimori, J. Phys. Soc. Jpn. 51, 3417 (1982)

[6] F.D.M. Haldane, J. Phys. C. 15, L1309 (1982)

[7] L.D. Faddeev, in Recent advances in field theory and statistical mechanics, Proceedings of the Les Houches Summer School 1982, edited by J.B. Zuber and R. Stora (North Holland, 1984)

[8] M. Toda, Theory of Nonlinear Lattices, Springer, Berlin (1981) and references cited therein

[9] N. Theodorakopoulos and N.C. Bacalis, Phys. Rev. B 46, 10706 (1992)

[10] N. Theodorakopoulos, Phys. Rev. B 30, 4071 (1984)

[11] J. Timonen, M. Stirland, D.J. Pilling, Yi Cheng and R.K. Bullough, Phys. Rev. Lett. 56, 2233 (1986)

[12] N-N. Chen, M.D. Johnson and M. Fowler, Phys. Rev. Lett. 56, 904 (1986)

[13] N. Theodorakopoulos and N.C. Bacalis, Phys. Rev. Lett. 67, 3018 (1991)

[14] N. Theodorakopoulos, unpublished (Ref. [14] in [13)

[15] M.E. Fisher, Am. J. Phys. 32, 343 (1964)

[16] M.J. Ablowitz and J.F. Ladik, J. Math. Phys. 17, 1011 (1976)

[17] H.M. Babujan, Phys. Lett. A 90, 479 (1982); L.A. Takhtajan. Phys. Lett. A 87, 479 (1982);

[18] N. Papanicolaou, J. Phys. A 20, 3637 (1987) 
[19] N. Theodorakopoulos, Phys. Lett. A 130, 249 (1988)

[20] N. Theodorakopoulos and E.W. Weller, Phys. Rev. B 37, 6200 (1988)

[21] K. Sasaki, Phys. Rev. B33, 2214 (1986)

[22] P. Schlottmann, Phys. Rev. Lett. 54, 2131 (1985); Phys. Rev. B 33, 4880 (1986)

[23] M. Takahashi and M. Yamada, J. Phys. Soc. Jpn. 54, 2808 (1985)

[24] R. Weber, Ph. D. Dissertation, University of Basel (1988)

[25] J.A. Krumhansl and J.R. Schrieffer, Phys. Rev. B 11, 3535 (1975)

[26] J.F. Currie, J.A. Krumhansl, A.R. Bishop and S.E. Trullinger, Phys. Rev. B 22, 477 (1980) 